\title{
Simple test for electrophysiologically tolerable parameters of artificial stimulation*
}

\author{
P. Bergveld \\ Twente University of Technology, PO Box 217, Enschede, The Netherlands
}

\begin{abstract}
Electrical stimulation of irritable tissue by a current source not only provides well defined stimulation but gives also the possibility to test whether the pulse amplitude, duration and rate are tolerable or not in order to prevent undesirable electrode reactions. This test can be very easily performed by recording the voltage developed between the stimulating electrodes as a function of time. This voltage must never reach constant value after a period of time.
\end{abstract}

Keywords-Artificial stimulation, Stimulation electrodes, Stimulation parameters

\section{Introduction}

WHEN DURING electrical stimulation of irritable tissue with certain values of current density and pulse duration, the stimulation does not cause a response, one is rapidly inclined to raise the total stimulation current or the pulse duration. In the following section it will be proved how far the current level and pulse duration can be raised before electrochemical reactions at the electrudes will disturb the physiological processes, and how easily the limiting values can be determined for a given electrode arrangement. It will be shown that stimulation with a current source is therefore preferred to stimulation with a voltage source.

\section{Polarisation of electrodes}

It is taken for granted that, in general, one can distinguish between reversible and irreversible electrodes. For electrical stimulation, inert irreversible electrodes are usually employed. This means that reactions occurring at the electrodeelectrolyte interface, result in electrical double layers, with corresponding potentials. The electrodes are polarised and it is likely that at the electrode surface chemical reaction products accumulate and even may separate from the solution (gasification) if certain values of stimulating current, pulse width and pulse frequency are exceeded. Because physiological solutions are in general well buffered and often assumed 'perfectly stirred' these electrode processes may not be detrimental to tissue but may certainly disturb the physiological processes at the place of stimulation. It is certain that many experiments where no attention was paid to these phenomena did not succeed, because gasification occurred at the site of stimulation. It is therefore obvious that in general this situation must be prevented and, as will be shown in this paper, the limiting values of the

\footnotetext{
"First received 25th April and in final form 25th September 1975
}

stimulating-current parameters can easily be established by considering the electrode potentials. These potentials are generally described as follows:

for the anode

$$
E_{A}^{\prime}=E_{A r e v}^{r}+\eta_{A} \quad \text {. . . . . . . }
$$

and for the cathode

$$
E_{c^{\prime}}=E_{C r e v}-\eta_{C} \quad . \quad . \quad . \quad . \quad . \quad . \quad .
$$

where $E_{A}{ }^{\prime}$ and $E_{C}{ }^{\prime}$ are the potentials of the anode and cathode, respectively, operating irreversibly; $E_{\text {Arev }}$ and $E_{C r e v}$ the reversible potentials and $\eta_{A}$ and $\eta_{C}$ the so called over-potentials. It will be obvious that the value of these over-potentials will be correlated with the state of the electrode processes. These processes

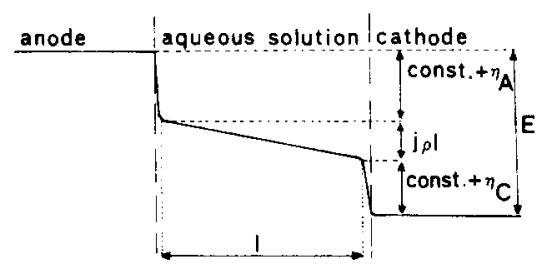

Fig. 1 Potential distribution between two metal electrodes in an aqueous solution with specific resistance $\rho$, during current flow with current density i (see text)

are determined by the reaction mechanism at the electrode-electrolyte interface which depends on the electrode material and the composition of the electrolyte. Nevertheless, they always result in an over potential and we can, for the purpose of investigating the limiting values of stimulation current, be concerned with the determination of the overpotentials.

A complete stimulation yields:

$$
E=E_{A}-E_{C}+j \rho l \text {. . . . . . . }
$$


where $E$ is the voltage between the two electrodes, $E_{A}$ the anode potential, which passes from $E_{A r e v}$ to $E_{A}{ }^{\prime}$ in a time which depends on the electrode processes at the anode, $E_{C}$ the cathode potential, which passes from $E_{\text {crev }}$ to $E_{C}{ }^{\prime}$ in a time which depends on the electrode processes at the cathode, $j$ the current density at the electrode surface, $\rho$ the specific resistance of the biological tissue which has to be stimulated and $l$ the length of the solution bulk between the electrodes, which is approximately the distance between the electrodes because the thickness of the double layers at the electrode surfaces may be the electrode processes. In a low overpotential region, the electrode reactions yield a linear relationship between the current density $j$ and the generated voltage $E$. In a somewhat higher overpotential region the voltage $E$ is a function of $\log I$ (so called Tafel behaviour). At still higher overpotentials the electrode reactions are diffusion controlled and the voltage $E$ is a function of $\sqrt{ } t$. At this state of the electrode reactions, an undesired accumulation of reaction products occurs, resulting for example in diatomic $\mathrm{H}_{2}$ or $\mathrm{O}_{2}$ evolution (gasification). This occurs after the adsorption of monotomic $\mathrm{O}$ or $\mathrm{H}$

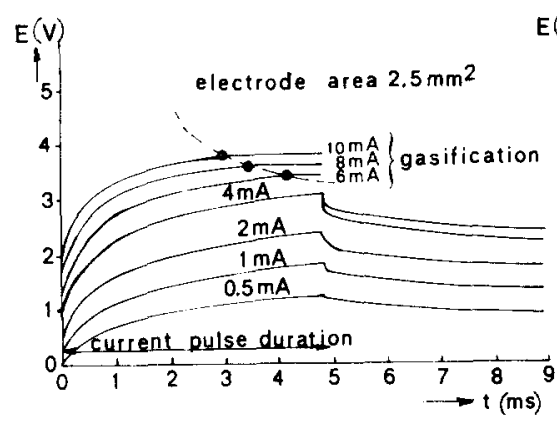

Fig. 2 Recordings of voltage curves, measured between stimulation electrodes which are supplied with a current pulse of $5 \mathrm{~ms}$ duration and 0.5-10 $\mathrm{mA}$ amplitudes

assumed to be very small. Combination of eqns. 1,2 and 3 gives

$$
\begin{aligned}
E & =E_{\text {Arev }}+\eta_{A}-\left(E_{\text {Crev }}-\eta_{C}\right)+j \rho l \\
& =E_{\text {Arev }}-E_{\text {Crev }}+\left(\eta_{A}+\eta_{C}\right)+j \rho l . . . .
\end{aligned}
$$

With $E_{\text {Aret }}-E_{\text {Crev }}$ is constant:

$$
E=\text { constant }+\left(\eta_{A}+\eta_{C}+j \rho l\right) \quad . \quad .
$$

This is shown in Fig. 1.

Therefore, between the electrodes a voltage is present which is the sum of the overpotentials and the potential drop across the volume of the electrolyte between the electrodes. This voltage $j \rho l$ is directly related to the transport of an electrical current through the solution, which will cause the depolarisation of a biomembrane, the aim of the electrical stimulation. However, this part of the total voltage $E$ across the stimulus electrodes is, in general, small compared with the overpotentials, generated at the electrodes. Therefore, if a voltage source is applied to the electrodes, the development of overpotentials will control the stimulation for the greater part, because the unknown overpotentials decrease the effective voltage considerably. However, using a current source for stimulation, the stimulation current is well defined and it appears that, measuring the corresponding overpotentials simultaneously as a function of time, one will be informed of the state of
The dashed line connects the points $E_{c r}, t_{c r}$, the points for which it is confirmed that gasification occurs when the pulse has a longer duration or higher amplitude than at this point

at the electrodes is saturated and after the buffering capacity of the physiological solution (local redox reactions) is exhausted, SCHULDINER and WARNER (1965). Because actual gasification has to be prevented, we will now only pay attention to this state of the electrode reactions, and thus to the region where the overpotentials are proportional to $\sqrt{ } t$ and the current density $j$.

In this case eqn. 5 yields

$$
\begin{aligned}
E & =\text { constant }+A j \sqrt{ } t+j \rho l \\
& =\text { constant }+j(A \sqrt{ } t+\rho l) \quad . \quad . \quad . \quad .
\end{aligned}
$$

in which $A$ is a function of the surface area and concentration of species. Thus the recorded potential

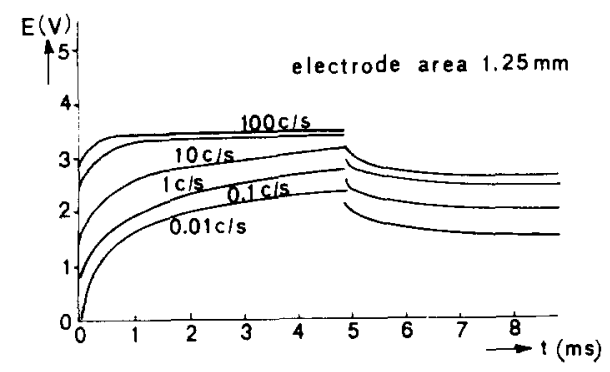

Fig. 3 Recording as Fig. 1b, with current pulse of $1 \mathrm{~mA}$ but with increasing stimulation frequency 
difference between the two electrodes during a constant current flow will increase with an increasing current density and with the time.

As mentioned above, as a criterion it is presumed that during stimulation no reaction products may leave the physiological solution under any circumstances. Therefore, it is assumed that a certain critical value of $E, E_{c r}$ and a certain critical value of time $t_{c r}$, may not be exceeded, so that care must be taken that a certain combination of current density and pulse duration is not exceeded.

\section{Experiments}

Although the electrode phenomena described accompany any irreversible electrode reactions, we shall only consider platinum electrodes, as these electrodes are general in use for electrical stimulation. As long as adsorption of monotomic gas occurs at the electrode surface a Platinum electrode behaves as a reversible electrode (and is in use as an oxygen sensor), SPECKMAN and CASPERS (1970), but as soon as absorption occurs with the evolution of diatomic gas molecules $\mathrm{H}_{2}$ and $\mathrm{O}_{2}$, the electrode process is irreversible and the above mentioned theory for irreversible electrode processes can be used.

During experiments with Platinum electrodes of different diameters in a Ringer solution, voltage curves were recorded during current pulses with different amplitudes and durations. Simultaneously, gasification was studied by means of a microscope. By slowly increasing the pulse duration or the pulse current density (by increasing the total current or by decreasing the electrode area in contact with the solution), it appears that gasification can only be observed with current pulses from which the corresponding voltage curves reach a constant value after a period of time. The point of a curve just before the first trace of gasification is visible by microscope is the critical combination of $E_{c r}$ and $t_{c r}$. In the practice of stimulation, this combination may never be exceeded. In Figs $2 a$ and $b$ the voltage curves are shown for two electrode areas, which are the results of supplying a current pulse with current values of 0.5 to $10 \mathrm{~mA}$ and a pulse duration of $5 \mathrm{~ms}$. In each curve, the point $\left(E_{c r}, t_{c r}\right)$ is given, being the point for which gasification can be observed for pulses which have a longer duration or a higher amplitude. The points $\left(E_{c r}, t_{c r}\right)$ are connected to each other by means of a dashed line, which obviously separates the horizontal parts and the nonhorizontal parts of the voltage curves. The dashed line shifts to the left for higher current densities (Fig. 2b).

Note that the falltime of the recorded voltage curves (when the current is switched off) has a much longer duration than the risetime. The reason for this is that during the current pulse, the electric current governs the ionic migration, while this only occurs by diffusion after the current is switched off. This phenomenon is called 'Faradic rectification', EYRING
(1970). During the recordings of Fig. 2, a pause was observed until the electrode potential had almost returned to zero before the next current pulse was generated. If this is neglected, a disturbance of the voltage curves as a function of the pulse interval time will also be observed, resulting in a shift of $\left(E_{c r}, t_{c r}\right)$ to lower values with decreasing interval time (increasing stimulation frequency). This is shown in Fig. 3 where the curves which correspond with a stimulation frequency of 10 and 100 pulses per second reach, after a period of time, a constant value. However, using the same current density per pulse but with a lower stimulation frequency the critical point $E_{c r}, t_{c r}$ is not exceeded.

From the recordings of Figs. 2 and 3, it can be concluded that stimulation with a certain pair of electrodes is only tolerable with combinations of current density, pulse duration and pulse rate, not exceeding the critical point $\left(E_{c r}, t_{c r}\right)$. In practice, this can be easily seen on the recorded voltage between the stimulation electrodes in the case of current stimulation. The voltage pulse must not reach a constant value. Increasing the current density or pulse rate forces a decrease in the pulse duration when a plateau in the voltage curve is observed. Increasing the pulse duration also forces a decrease in the current density or pulse rate as soon as a plateau in the voltage curve is observed. If stimulating a nerve or muscle fibre with an arbitrary current and pulse duration does not result in a contraction, while the voltage curve marginally is beginning to reach a plateau, the stimulation electrodes have to be inserted nearer to the nerve or muscle fibre to be excited, or bigger electrodes (larger area) have been used in order to be able to increase the total stimulation current.

Summarising, it may be concluded that stimulation by means of a current source can be well defined and also provides a possibility for testing the tolerable values of the pulse amplitude, duration and rate. This test can easily be performed during the stimulation by recording the voltage developed between the electrodes by the current pulse. To ensure a minimal molecular gas generation or other objectionable irreversible reactions, this voltage must never reach a constant value.

Acknowledgment-The author wishes to thank $\mathrm{H}$. Meertens for carrying out the measurements accurately.

\section{References}

EYRING, H. (1970) Physical Chemistry, An advanced treatise. 9 vol. A /Electrochemistry. chap. 4, Techniques for the study of Electrode processes. Academic Press.

SCHULDINER, S., and WARNER, B. (1965) Investigations of the kinetic of hydrogen and oxygen reactions on a platinum electrode in acid solution using pulse and decay techniques. J. Electrochem. Soc. 112, 212-218. 
Speckman, E. J., and Caspers, H. (1970) Messung des Sauerstoffdrucks mit platin Mikroelektroden in zentral nerven System. Pflugers Arch. 318, 78-84.

\section{Other Literature}

Greatbatch, W., and Chardack, W. M. (1968) Myocardial and endocardiac electrodes for chronic implantations. Bioelectrodes. Amn. N. Y. Acad. Sci. 148, 234-251.

MANSFIELD, P. B. (1967) Myocardial stimulation: the electrochemistry of electrode-tissue coupling. $\mathrm{Am} . \mathrm{J}$. Physiol. 212, 1475-1488.

Weinman, J., and MaHLer, J. (1964) An analysis of electrical properties of metal electrodes. Med. Electron. Biol. Engng. 2, 299-310. 